\title{
Temperature Estimation in a Magneto-Rheological Damper
}

\author{
Gianluca Savaia ${ }^{1}$, Matteo Corno ${ }^{1}$, Giulio Panzani $^{1}$, Andrea Sinigaglia ${ }^{2}$, Sergio M. Savaresi ${ }^{1}$
}

\begin{abstract}
Magneto-rheological dampers are employed in the automotive industry to control the vehicle dynamics by modulating the damping characteristics of the suspension system; these devices rely on a smart fluid which can change its viscosity when subjected to a magnetic field. The viscosity of this magneto-rheological fluid is significantly dependent on the operating temperature; this phenomenon is particularly critical in the automotive field since the working conditions span a wide range of temperatures and, furthermore, a commercial vehicle cannot be equipped to directly measure the temperature of the fluid. This article proposes a methodology for the temperature estimation which exploits the thermodynamic relationship between the resistance of the electrical circuit of the device and the temperature of the magneto-rheological fluid.
\end{abstract}

\section{INTRODUCTION}

Magneto-rheological (MR) dampers are a powerful mean for controlling the vehicle vertical movements and vibrations induced by the road excitation [1]. These devices, differently from electro-hydraulic suspensions, employ MR fluid to generate friction, which can change its viscosity with respect to the magnetic field it is subjected to.

It is well known in literature that the viscosity of the MR fluid is significantly affected by the operating temperature [2], [3], [4] diminishing the damping capabilities of the MR damper [5], [6]. As remarked in [7], since the MR damper dissipates the vehicle mechanical energy, the heating of the internal damper fluid cannot be avoided. There has been research in the optimal geometry for a MR damper with the aim of minimizing the heat transfer to the MR fluid [8], [9]; nonetheless, the studies conclude that the effect of temperature cannot be neglected.

For these reasons, it is important to include a compensation of this effect in the control strategies that exploit such devices. In [10], [11], the authors investigate the effect of the temperature on the performance and the stability of feedback controllers; in [12], the problem is framed in a fault-tolerant approach by adopting a sliding mode controller. Further research has focused on the possibility to compensate the performance decay with neural networks [13], [14]. All of the above control frameworks assume the temperature is measurable, which is not often the case, especially in commercial vehicles. Hence, the need to estimate the temperature of the MR fluid.

The most common approach which can be found in literature to solve this task makes use of a thermal model of the MR damper, as developed e.g. in [15] where the model describes the heat exchange between the electromagnet and

\footnotetext{
${ }^{1}$ Dipartimento di Elettronica, Informazione e Bioingegneria, Politecnico di Milano, Milano, Italy. matteo.cornoepolimi.it

${ }^{2}$ Automobili Lamborghini, Sant'Agata Bolognese, Italy.
}

the MR fluid. The model has also been validated in [16] where the authors also indicate that both mechanical and electrical power input contribute to the fluid temperature rise. In a similar research [17], the authors developed a complex thermal model for an automotive twin-tube shock absorbers. Further work exploits a neural network for the estimation of the damping force with a variable temperature operation [18]. These methodologies exploiting a thermal model suffer from robustness and complexity, since a detailed description of both geometry and heat transfer coefficient must be characterized. Further, the heat exchange is also dependent on the temperature of the environment, operating conditions of the vehicle (e.g., a high traveling speed can cool down quickly the dampers) and the location in the vehicle body (e.g., the distance of the damper from the engine).

This work proposes a different approach for the estimation of the MR fluid temperature without the need to develop a thermal model, under the assumption that the temperature of the coil generating the magnetic field and the temperature of the MR fluid are similar; this is generally true when considering a classic geometry of a MR damper, where the coil is placed within the piston which, in turn, is submerged in the MR fluid. Being the electric resistance of the coil known to be to be proportional to the temperature of the conductor, we are then able to infer the temperature of the MR fluid at any given time by properly analyzing the available electric measures. The proposed approach is particularly suited for the application in the automotive field where the dampers' temperature is not directly measurable and, to the authors' knowledge, this is the first work where this methodology is proposed for a MR damper.

Two strategies, both based on the aforementioned idea, are discussed, compared and validated with experimental data: a piecewise strategy which gives a more accurate estimation but requires an ad hoc current injection into the MR damper's coil, and a continuous strategy which can estimate the temperature without interfering with the underlying suspension control strategy.

The remainder of this paper is as follows. In Section II, the basic principles which this research is based upon are presented. The experimental setup employed to characterize the relationship between resistance and temperature is elaborated in Section III and the results are presented in Section IV. The estimation strategies are described in Section $\mathrm{V}$ and validated quantitatively in Section VI. The paper ends with some concluding remarks. Throughout the paper, for confidentiality reasons, the axes of some Figures have been normalized. 


\section{PROBLEM STATEMENT}

The magneto-rheological fluid can change its viscosity according to the magnetic field which is subjected to. The magnetic field is generated within the piston's head by the current flowing into an electromagnetic coil, which is controlled via the voltage potential applied to the ends of the coil itself. Therefore, it is possible to regulate the fluid's viscosity via the input control current.

However, the viscosity is also influenced by the working temperature of the fluid: the lower the temperature, the higher the damping forces exerted by the damper. Automotive applications consider a possible temperature range between $-30{ }^{\circ} \mathrm{C}$ and $+140{ }^{\circ} \mathrm{C}$; as shown in [5], [6], with such variations the effect of the temperature on the MR fluid viscosity cannot be ignored.

The aim of this research consists in estimating the temperature of the MR fluid by exploiting its correlation with the temperature of the coil used as proxy. This assumption holds true, in general, since the piston's head is immersed in the fluid. However, the temperature of the coil is not directly measurable either, and needs to be inferred via the estimation of the coil's electric resistance.

Indeed, the coil in the piston can me modeled as a RL circuit described by the equation

$$
L \frac{d i}{d t}+R i=V
$$

where $R$ and $L$ are the resistance and inductance of the coil, $i$ the current flowing into it and $V$ the applied voltage; considering a steady-state scenario, equation (1) simply becomes

$$
R=\frac{V}{i}, \quad i \neq 0 .
$$

Eventually, it is well known in literature [19] that the resistivity $\rho$ of a conductor is related to its temperature

$$
\rho=\rho_{0}\left[1+\alpha\left(T-T_{0}\right)\right] .
$$

It is thus clear how the estimate of the MR fluid temperature will be done via the estimation of the coil's resistance
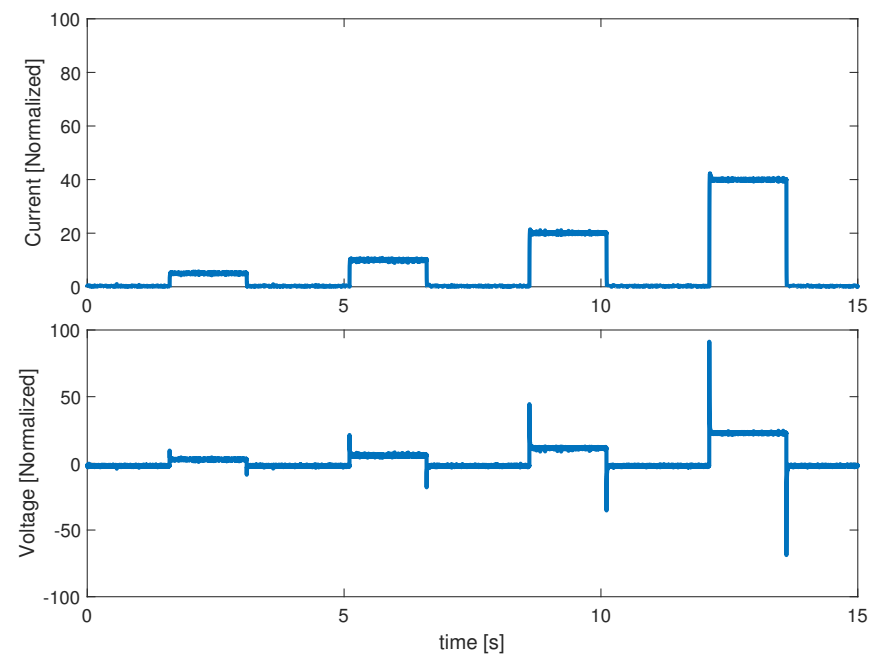

Fig. 1. Current profile in the stairs experiment. in the piston of the damper, based on the available electric measures $i$ and $V$.

\section{EXPERIMENTAL SETUP AND TESTS}

The proposed strategies have been developed using the data collected during an experimental campaign. The MR dampers installed on the vehicle have been additionally instrumented with thermocouples which allow to measure directly the temperature of the MR fluid inside the chamber, and are used to assess the estimation performance.

Each MR damper is powered by a $12 \mathrm{~V}$ battery and controlled by an Electronic Control Unit (ECU) implementing a current control loop via Pulse Width Modulation (PWM). Hence, in this configuration, it is possible to command a reference current to be fed to the coil inside the piston's head. The actual voltage potential $V$ applied to the coil is available for measurement from the ECU.

The vehicle is driven on a rough road in order to excite the vertical dynamics causing the MR damper heating; the temperature increase transient depends on the ambient conditions and the road excitation. The tests are stopped when the dampers reach the maximum allowed working temperature. An example of temperature profile recorded during an experiment is shown in Fig. 3, where the damper suffered a temperature excursion of $60 \%$ with respect to its working range in 40 minutes (the actual value is normalized for confidentiality reasons).

During the experiments, two different current profiles have been designed: stairs and ping, depicted in Fig. $1-2$ respectively. The stairs experiment is designed ad hoc to collect more information on the effect of the current in the estimation of the coil's resistance, which is investigated in Section IV. The ping experiment, on the other hand, resembles a real scenario where the temperature estimation and suspension control are operated concurrently, as shown in Fig. 2 where the constant current ping overrides the control logic.

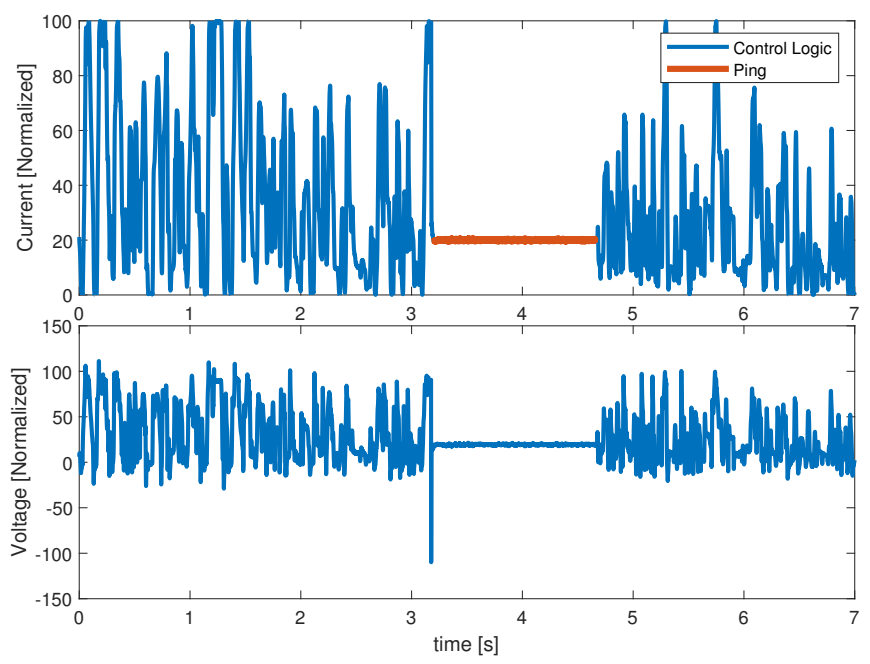

Fig. 2. Current profile in the ping experiment. 


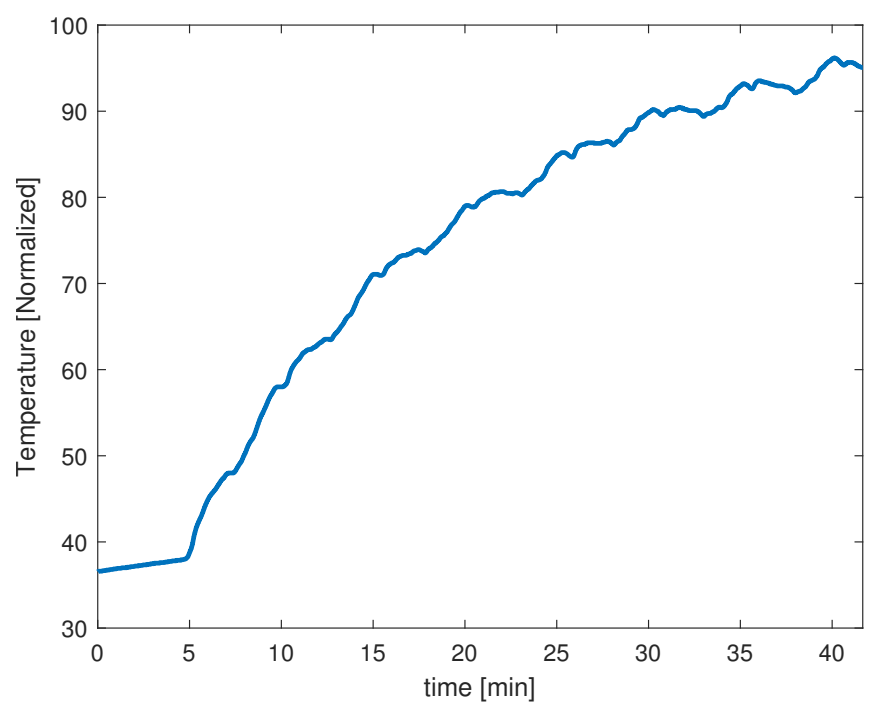

Fig. 3. Measured temperature of the MR fluid during the experiment.

In the design of the experiment, it's important to recall that the amplitude of the current shall not reach the maximum current for long time spans, in order to avoid heating the coil due to the steady high current flow; this phenomenon would break the assumption elaborated in Section II, since the MR fluid and the coil would not be at the same temperature. For this reason, in both experiments the ping is never higher than $50 \%$ of the maximum possible current.

The ECU can record all the signals of interest at a sampling frequency of $1 \mathrm{kHz}$; these data are then postprocessed and analyzed offline.

\section{PRELIMINARY DATA ANALYSIS}

In this section, the analysis of the stairs experiment is firstly presented, since the estimation strategies will build upon these observations.

The relationship in (2) is only valid when the current flowing in the coil is constant and different from zero; hence, the estimation of the resistance is computed by extracting those segments (taking care of excluding the transient behaviors) and averaging the value of the measured current $i$ and voltage $V$ as in

$$
\bar{R}=\frac{\bar{V}}{\bar{i}}
$$

where $\bar{V}$ is the average voltage, $\bar{i}$ the average current and $\bar{R}$ is the resulting estimated resistance in the segment.

Let $\bar{T}$ be the average measured temperature computed in each corresponding steady-state segment; then, the sought relationship between temperature and resistance can be mapped on the plane depicted in Fig. 4 for the stairs experiment. A clear linear relationship between the two quantities is visible, which can be modeled by

$$
\bar{T}=\alpha \bar{R}+T_{0}(i),
$$

where $\alpha$ indicates the slope, and $T_{0}$ the offset which, as can be seen from the figure, depends on the current injected into the coil. This varying bias is not due to a temperature effect,

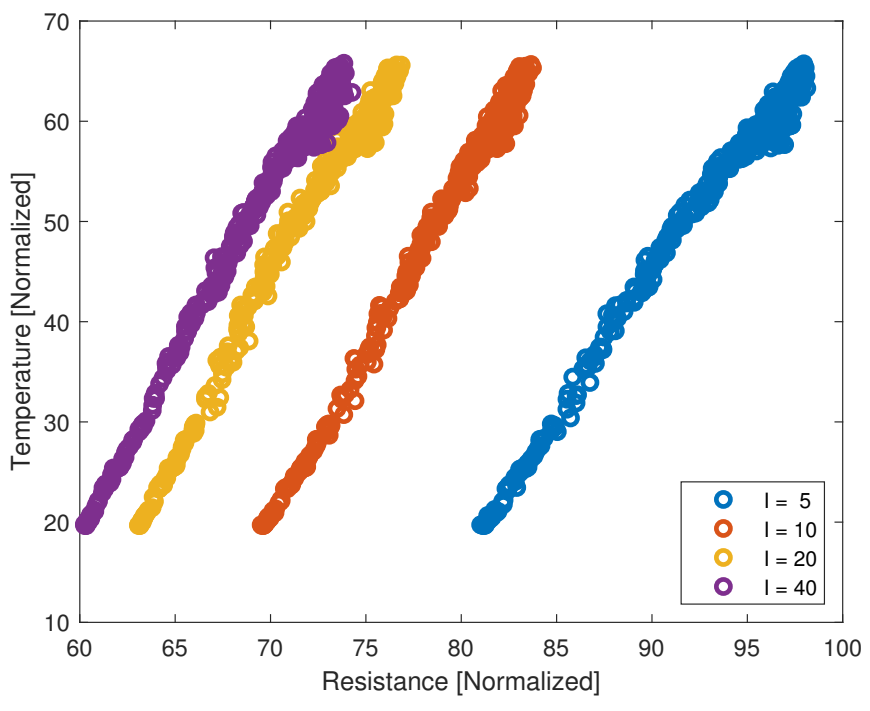

Fig. 4. Temperature - Resistance map for the stairs experiment.

since a higher current flow should lead to a higher resistance of the conductor, whereas in Fig. 4 the opposite happens. Hence, the only plausible conclusion is the relationship between current and voltage, in our scenario, is not constant as in (2).

Therefore, given any constant value for the current amplitude (different from zero), the relationship between MR fluid's temperature and coil's resistance is well defined. In the following section, this relationship is exploited to design the estimation strategies.

\section{TEMPERATURE ESTIMATION STRATEGIES}

Considering the linear regression suggested by the experimental data in Fig. 4, given a resistance value $R$ the temperature MR fluid temperature is computed as in (4).

Two different strategies are presented to compute the value of $R$ based on the available measures: in particular the piecewise strategy requires a current ping, whereas the continuous estimation is capable of providing a value of $R$ without interfering with the suspension control strategy.

\section{A. Piecewise Estimation}

This strategy is based upon the ping experiment, shown in Fig. 2, where the suspension control is overridden by a constant current ping. It is important to notice that, although the effect should be minimal, this methodology perturbs the control logic.

When the ping is raised, the time series of the signals $i(t)$ and $V(t)$ are collected (excluding the transients) and averaged in order to compute $\bar{R}$ as in (3). Eventually, the temperature $\hat{T}$ is estimated as in (4), where the parameters $\alpha$ and $T_{0}$ represent the model and their value is the solution to the least squares optimization

$$
\left\|\bar{T}-\left(\alpha \bar{R}+T_{0}\right)\right\|_{2} .
$$

It is worth to remark that, once the ping amplitude has been chosen, $T_{0}$ is a constant. The results of this approach 


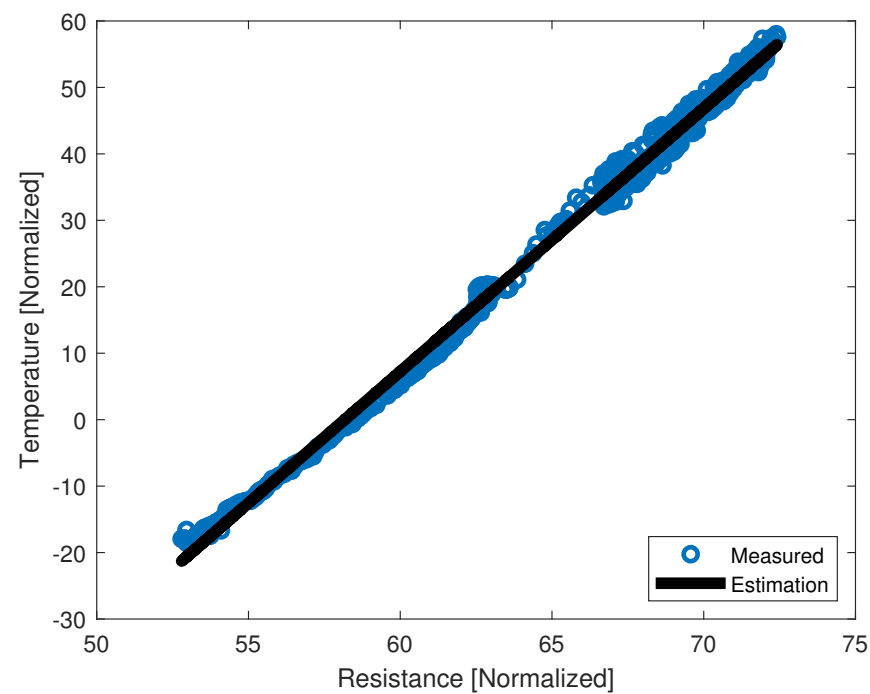

Fig. 5. Temperature - Resistance regression for the piecewise strategy.

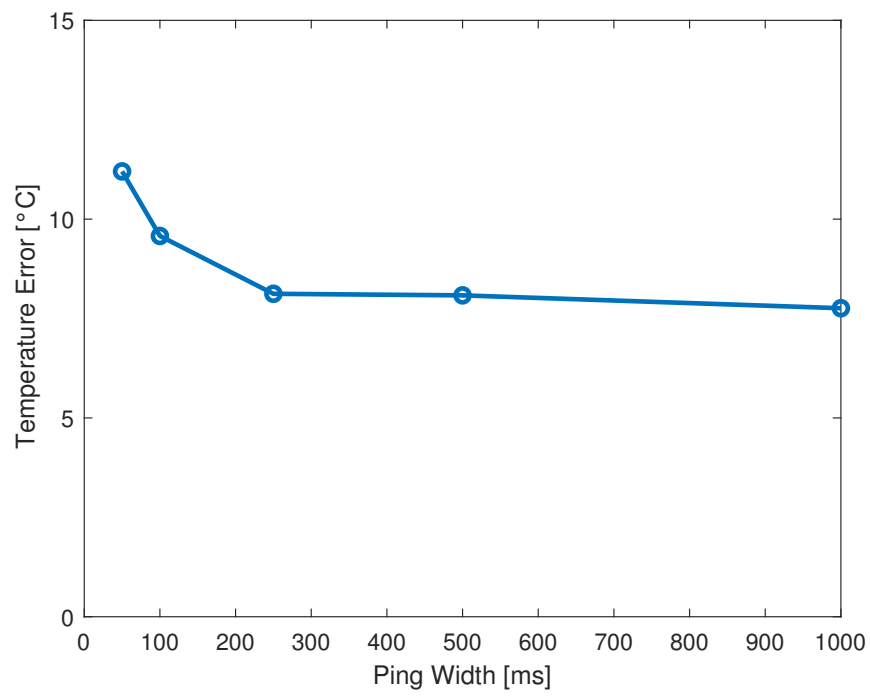

Fig. 6. Sensitivity of the maximum estimation error with respect to the ping duration.

are depicted in Fig. 5 and a quantitative evaluation of the methodology is elaborated in Section VI.

The experiments in Fig. $1-2$ are based on the injection of a ping of width $1500 \mathrm{~ms}$. However, since the ping is an undesired action which may affect the vehicle dynamics, this duration may be too long for our purposes; hence, a sensitivity analysis with respect to the ping duration is shown in Fig. 6: values below $250 \mathrm{~ms}$ yield a poor accuracy due to measurement noise, whereas higher ones do not improve the estimation performance significantly. Therefore, $250 \mathrm{~ms}$ is the optimal compromise between estimation accuracy and perturbation of the control logic.

\section{B. Continuous Estimation}

The second methodology proposed is based upon the relationship (2) which is valid in steady-state condition only; since this state is never realized when the suspension control logic is working, in this approach the signals are low-pass filtered in order to meet that condition.

Let $L_{\tau}$ be a first order filter described by the transfer function

$$
L_{\tau}(s)=\frac{\frac{2 \pi}{\tau}}{s+\frac{2 \pi}{\tau}}
$$

where the filter is parametrized as function of the time constant $\tau$, which gives an approximation of the filter's settling time.

Therefore, the estimation of the coil's resistance is computed continuously as

$$
R_{\tau}(t)=\frac{V_{\tau}(t)}{i_{\tau}(t)}
$$

where $i_{\tau}$ and $V_{\tau}$ are the outputs of the filter $L_{\tau}(s)$. This relation trivially holds so long as $i_{\tau} \neq 0$.

The choice of $\tau$ consists in a trade-off between estimation accuracy and perturbations. Ideally, a high value of $\tau$ implies a low bandwidth which is close to the assumption of steady-state condition; on the other hand, it implies a slower response causing the estimation to lag behind the measured value. In Fig. 7, a lower value for $\tau$ shows a better tracking of the measured temperature, whilst yielding a poor estimation of the coil's resistance, which in turn it reflects on an oscillating temperature estimation.

As it is possible to observe in Fig. 8, the linear relationship between coil's resistance and MR fluid's temperature still holds, even though visibly less accurate. The two depicted experiments share the same slope with a different bias which is due to the different current profile injected into the damper; this effect is similar to the observations discussed in Section IV where the dependency between the resistance estimation with respect to the injected current has been outlined.

Whereas in the piecewise strategy the bias effect could be worked around by injecting a known constant ping, in the continuous strategy the current profile cannot be assumed to be known. Thus, following this approach, the regression parameter $T_{0}$ in (4) cannot be considered constant, but shall
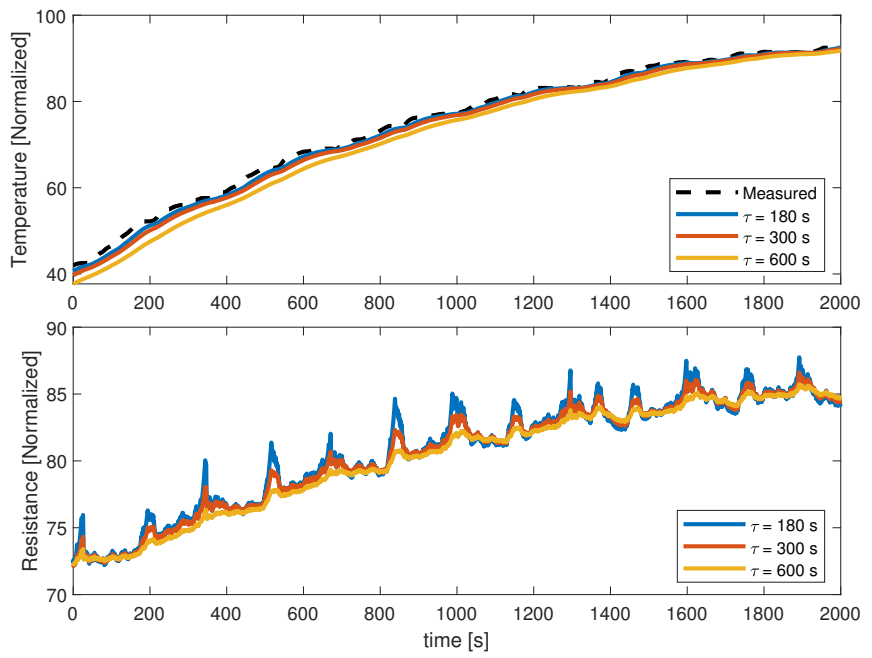

Fig. 7. Sensitivity of the estimation with respect to $\tau$. 


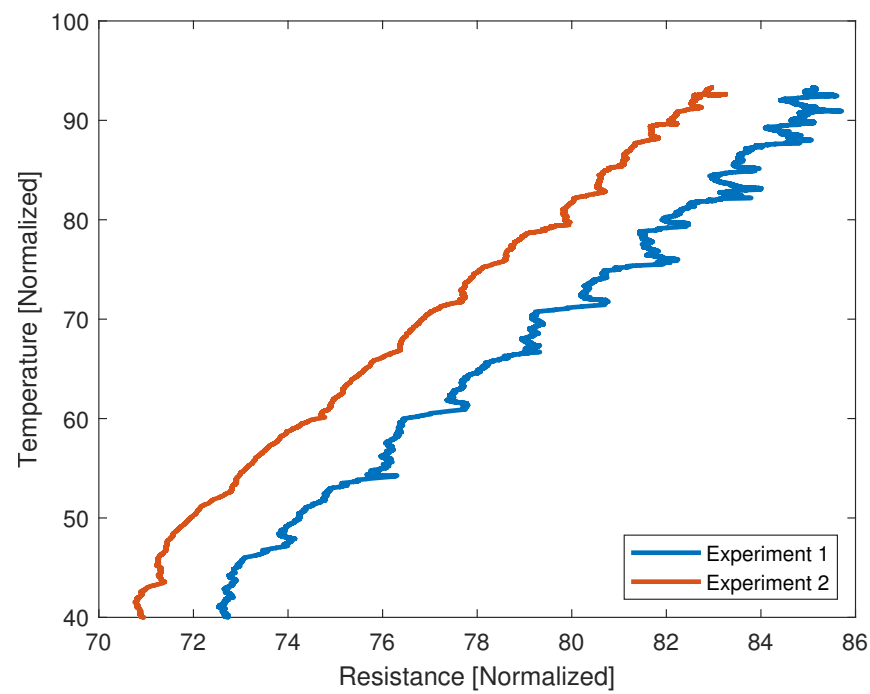

Fig. 8. Temperature - Resistance relationship in the continuous strategy for two experiments in different conditions.

be parametrized with respect to the filtered current. Hence, the temperature estimation $\hat{T}$ becomes

$$
\hat{T}(t)=\alpha R_{\tau}(t)+T_{0}\left(i_{\tau}(t)\right)
$$

The relationship between $T_{0}$ and $i_{\tau}$ is drawn from the stairs experiment, in Fig. 4, where the dependency is clearly portrayed. In Fig. 9, the case where $T_{0}$ is assumed to be constant is compared against the compensation strategy; it is shown how the compensation mitigates the bias observed in Fig. 8 drastically improving the estimation error, even though the accuracy is significantly worse than the previous method, as it is thoroughly elaborated in Section VI.

The poorer performance can be explained by the following rationales:

- the steady-state condition in (2) is not met, although the low-pass filtering is a good approximation of that configuration;

- when the current $i_{\tau}$ is small, (2) approaches its singularity point and the estimation is less robust.

\section{VALIDATION}

In this section, the validation of the estimation strategies presented in Section $\mathrm{V}$ is performed on different experiments performed in diverse conditions (e.g., weather condition, test road). With the aim of assessing quantitatively the experiments and comparing the two approaches, two sets of error indexes are defined: one for the piecewise strategy

$$
\begin{aligned}
& \varepsilon_{r m s}=\sqrt{\frac{1}{N} \sum_{k=0}^{N}(\bar{T}-\hat{T})(k)} \\
& \varepsilon_{\max }=\max \|\bar{T}(k)-\hat{T}(k)\|_{1}
\end{aligned}
$$

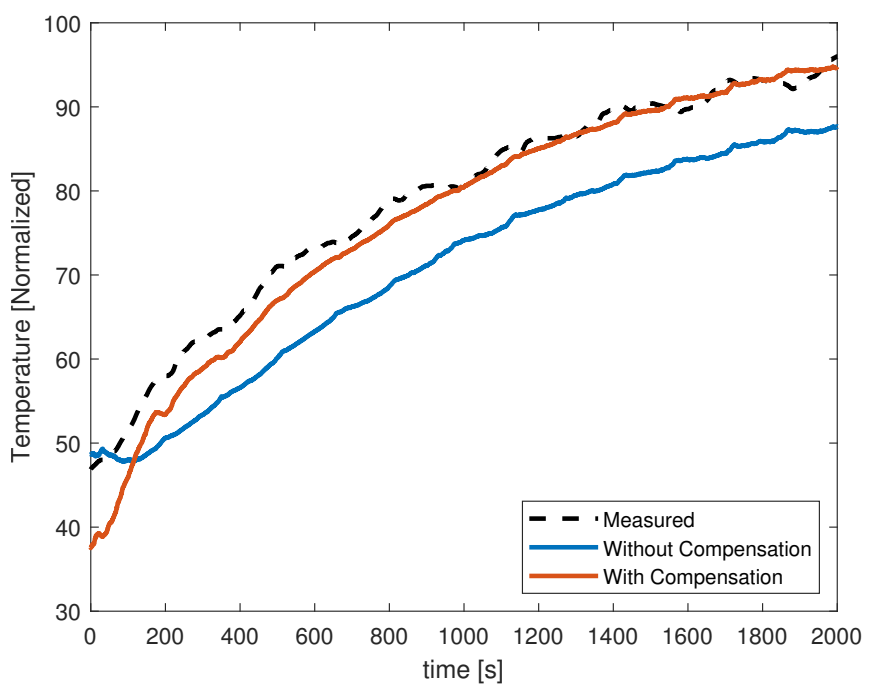

Fig. 9. Temperature estimation with the continuous strategy.

and the other set for the continuous strategy

$$
\begin{aligned}
\varepsilon_{r m s} & =\sqrt{\frac{1}{N} \int_{t=0}^{N}(T-\hat{T})(t)} \\
\varepsilon_{\max } & =\max \|T(t)-\hat{T}(t)\|_{1}
\end{aligned}
$$

It is worth remarking that the former refers to a time series where each instant corresponds to a ping of current, whereas the latter is a continuous time evaluation of the error; observe that, although the indexes are computed differently, the comparison between the two set of error indexes is fair.

The estimation strategies proposed have been validated in the scenario described in Section III; in order to evaluate the efficacy of the strategies in a real case study, the current profile fed to the MR damper follows the ping experiment in Fig. 2, where the suspension control logic is overridden by a ping of constant current (the ping is only needed by the piecewise strategy, whereas the continuous strategy ignores it).

The results are presented in Fig. $10-11$, where each experiment is characterized by a different current profile. The piecewise strategy is the most accurate, with a maximum error of $6{ }^{\circ} \mathrm{C}$, whereas the continuous strategy yields, in general, much poorer results. In particular, the latter strategy performs badly in those experiments where the average current is low $(<300 \mathrm{~mA})$, confirming the statements at the end of Section V-B.

\section{CONCLUSIONS}

This article proposes two strategies, piecewise and continuous, for the estimation of the temperature of the MR fluid contained in a MR damper, presenting the theoretical foundations for the viability of estimating the temperature through the resistance of the coil inside the piston.

The first strategy is based on the injection of a constant current (i.e., ping) into the coil, which allows the algorithm to estimate the temperature in the time span of the ping. 


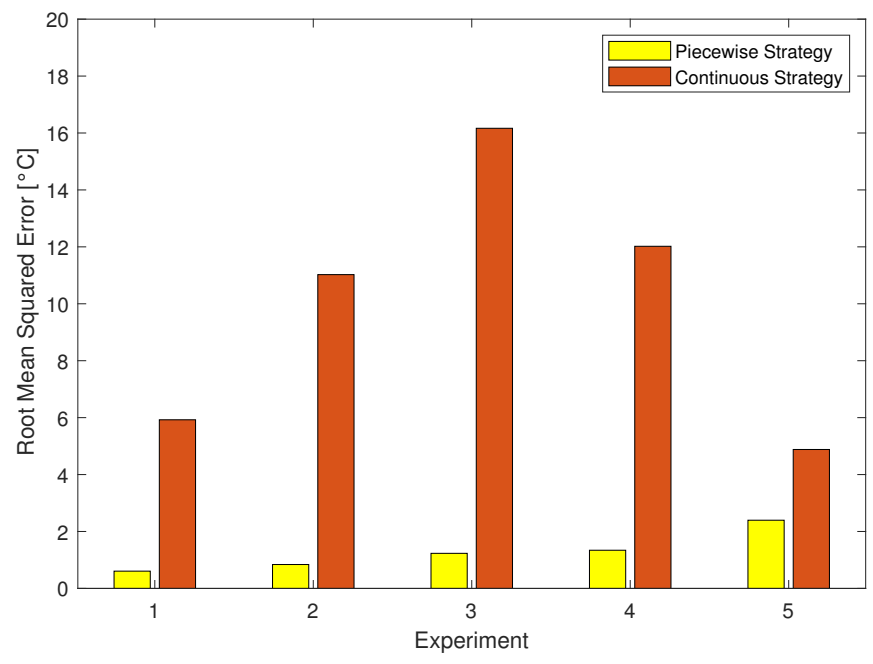

Fig. 10. Validation of the estimation strategies over 5 experiments using the root mean squared error $\varepsilon_{r m s}$.

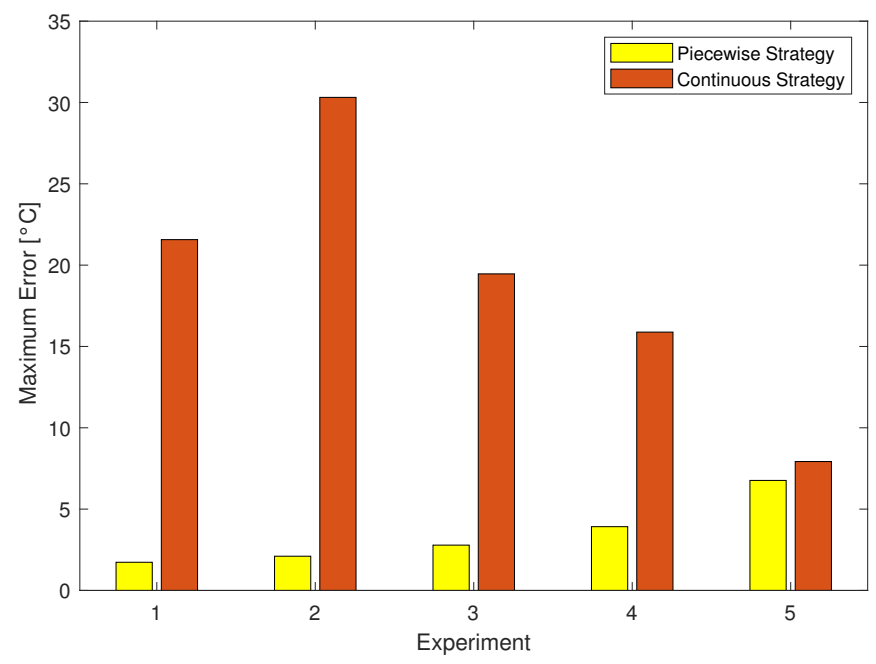

Fig. 11. Validation of the estimation strategies over 5 experiments using the maximum error $\varepsilon_{\max }$.

The latter strategy can yield a continuous estimate of the temperature without needing any ping.

The results, which have been validated on a real set-up, show the piecewise strategy gives a more accurate estimation of the MR fluid's temperature $\left( \pm 6{ }^{\circ} \mathrm{C}\right)$, whereas the continuous strategy performs much worse $\left( \pm 20^{\circ} \mathrm{C}\right)$.

The piecewise strategy is better suited for those applications where a higher accuracy is requested and it is possible to override the current with a ping signal; on the contrary, when it is not possible to inject a ping, the continuous strategy shall be employed to estimate the temperature of the MR fluid in a MR damper.

Eventually, the proposed strategies are not computationally burdensome; hence, they can be deployed to any on-board ECU for a real-time estimation.

\section{ACKNOWLEDGMENT}

The authors thank Automobili Lamborghini for providing the magneto-rheological damper, the vehicle, and for helping to carry out the experiments.

\section{REFERENCES}

[1] D. Batterbee and N. Sims, "Vibration isolation with smart fluid dampers: a benchmarking study," Smart Structures and Systems, vol. 1, no. 3, pp. 235-256, 2005.

[2] S. G. Sherman, L. A. Powell, A. C. Becnel, and N. M. Wereley, "Scaling temperature dependent rheology of magnetorheological fluids," Journal of Applied Physics, vol. 117, no. 17, p. 17C751, 2015.

[3] H. Sahin, X. Wang, and F. Gordaninejad, "Temperature dependence of magneto-rheological materials," Journal of Intelligent Material Systems and Structures, vol. 20, no. 18, pp. 2215-2222, 2009.

[4] Y. Rabbani, M. Ashtiani, and S. H. Hashemabadi, "An experimental study on the effects of temperature and magnetic field strength on the magnetorheological fluid stability and mr effect," Soft matter, vol. 11, no. 22 , pp. 4453-4460, 2015.

[5] M. J. McKee, Effects of temperature on performance of compressible magnetorheological fluid dampers. University of Nevada, Reno, 2010.

[6] N. L. Wilson, N. M. Wereley, W. Hu, and G. J. Hiemenz, "Analysis of a magnetorheological damper incorporating temperature dependence," International Journal of Vehicle Design, vol. 63, no. 2-3, pp. 137-158, 2013, pMID: 56102 .

[7] M. Alonso and Á. Comas, "Thermal model of a twin-tube cavitating shock absorber," Proceedings of the Institution of Mechanical Engineers, Part D: Journal of Automobile Engineering, vol. 222, no. 11, pp. 1955-1964, 2008.

[8] X. Dong, J. Yu, W. Wang, and Z. Zhang, "Robust design of magnetorheological (mr) shock absorber considering temperature effects," The International Journal of Advanced Manufacturing Technology, vol. 90, no. 5-8, pp. 1735-1747, 2017.

[9] M. M. Ferdaus, M. Rashid, M. H. Hasan, H. B. M. Yosuf, M. Bhuiyan, and A. Alraddadi, "Temperature effect analysis on magneto-rheological damper's performance," Journal of Automation and Control Engineering Vol, vol. 2, no. 4, 2014.

[10] D. Batterbee and N. D. Sims, "Temperature sensitive controller performance of mr dampers," Journal of Intelligent Material Systems and Structures, vol. 20, no. 3, pp. 297-309, 2009.

[11] D. Batterbee and N. Sims, "Temperature sensitive stability of feedback controllers for mr dampers," in Active and Passive Smart Structures and Integrated Systems 2008, vol. 6928. International Society for Optics and Photonics, 2008, p. 69281B.

[12] X. Dong, M. Yu, and Z. Guan, "Adaptive sliding mode faulttolerant control for semi-active suspension using magnetorheological dampers," Journal of Intelligent Material Systems and Structures, vol. 22, no. 15, pp. 1653-1660, 2011.

[13] H. Metered, P. Bonello, and S. Oyadiji, "The experimental identification of magnetorheological dampers and evaluation of their controllers," Mechanical systems and signal processing, vol. 24, no. 4, pp. 976-994, 2010.

[14] H. Metered, P. Bonello, and Oyadiji, "An investigation into the use of neural networks for the semi-active control of a magnetorheologically damped vehicle suspension," Proceedings of the Institution of Mechanical Engineers, Part D: Journal of Automobile Engineering, vol. 224, no. 7, pp. 829-848, 2010.

[15] F. Gordaninejad and D. G. Breese, "Heating of magnetorheological fluid dampers," Journal of intelligent material systems and structures, vol. 10, no. 8, pp. 634-645, 1999.

[16] M. B. Dogruoz, E. L. Wang, F. Gordaninejad, and A. J. Stipanovic, "Augmenting heat transfer from fail-safe magneto-rheological fluid dampers using fins," Journal of Intelligent Material Systems and Structures, vol. 14, no. 2, pp. 79-86, 2003.

[17] J. Ramos, A. Rivas, J. Biera, G. Sacramento, and J. Sala, "Development of a thermal model for automotive twin-tube shock absorbers," Applied Thermal Engineering, vol. 25, no. 11-12, pp. 1836-1853, 2005.

[18] A. Patel and J. Dunne, "Narx neural network modelling of hydraulic suspension dampers for steady-state and variable temperature operation," Vehicle system dynamics, vol. 40, no. 5, pp. 285-328, 2003.

[19] M. Ward, Electrical Engineering Science. McGraw-Hill, 1971, pp. $36-40$. 